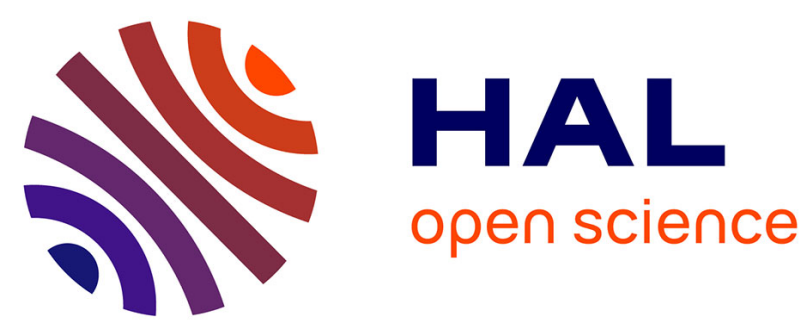

\title{
Direct approach-based analysis of plates composed of functionally graded materials
}

\author{
Holm Altenbach, Victor A. Eremeyev
}

\section{To cite this version:}

Holm Altenbach, Victor A. Eremeyev. Direct approach-based analysis of plates composed of functionally graded materials. Archive of Applied Mechanics, 2008, 78 (10), pp.775-794. hal-00823985

\section{HAL Id: hal-00823985 \\ https://hal.science/hal-00823985}

Submitted on 20 May 2013

HAL is a multi-disciplinary open access archive for the deposit and dissemination of scientific research documents, whether they are published or not. The documents may come from teaching and research institutions in France or abroad, or from public or private research centers.
L'archive ouverte pluridisciplinaire HAL, est destinée au dépôt et à la diffusion de documents scientifiques de niveau recherche, publiés ou non, émanant des établissements d'enseignement et de recherche français ou étrangers, des laboratoires publics ou privés. 
Holm Altenbach • Victor A. Eremeyev

\title{
Direct approach-based analysis of plates composed of functionally graded materials
}

\begin{abstract}
The classical plate theory can be applied to thin plates made of classical materials like steel. The first theory allowing the analysis of such plates was elaborated by Kirchhoff. But this approach was connected with various limitations (e.g., constant material properties in the thickness direction). In addition, some mathematical inconsistencies like the order of the governing equation and the number of boundary conditions exist. During the last century many suggestions for improvements of the classical plate theory were made. The engineering direction of improvements was ruled by applications (e.g., the use of laminates or sandwiches as the plate material), and so new hypotheses for the derivation of the governing equations were introduced. In addition, some mathematical approaches like power series expansions or asymptotic integration techniques were applied. A conceptional different direction is connected with the direct approach in the plate theory. This paper presents the extension of Zhilin's direct approach to plates made of functionally graded materials.
\end{abstract}

Keywords Functionally graded materials · Plate theories - Direct approach · Effective stiffness · Transverse shear stiffness

\section{Introduction}

The Kirchhoff's plate theory suggested more than 150 years ago [18] was worked out for thin plates made of homogeneous isotropic materials and the case of bending. Introducing the deflection of the midplane the theory is limited by the assumption that each point of the midplane has one independent degree of freedom. Finally, the governing equation for the bending problem can be deduced as

$$
\Delta \Delta w=\frac{q}{D}
$$

with the deflection $w$, the surface load $q$, the Laplacian $\Delta$ and the bending stiffness $D$. In the background of this theory, one can see for engineering applications very fruitful assumptions: instead of the stresses stress resultants (averaged stresses in the thickness direction) are introduced - the forces and the moments-which have a clear mechanical interpretation, and the individual mechanical properties can be presented by another

The second author was supported by DFG grant 436RUS17/21/07.

H. Altenbach $(\bowtie)$

Lehrstuhl für Technische Mechanik, Zentrum für Ingenieurwissenschaften, Martin-Luther-Universität Halle-Wittenberg, 06099 Halle (Saale), Germany

E-mail: holm.altenbach@iw.uni-halle.de

\section{A. Eremeyev}

South Scientific Center of RASci and South Federal University, Milchakova str. 8a, 344090 Rostov on Don, Russia E-mail: eremeyev@math.rsu.ru 
averaged property-the bending stiffness which is an effective property combining the Young's modulus $E$, the Poisson's ratio $v$ and the thickness $h$

$$
D=\frac{E h^{3}}{12\left(1-v^{2}\right)}
$$

On the other hand one observes very rough limitations which are not helpful in modern applications. The following are examples of such limitations:

- The thinness hypotheses,

- The isotropy assumption,

- The homogeneity assumption,

- The small deflection assumption,

- The assumption that the plate behavior (out-of-plane) and the plane stress behavior (in-plane) are decoupled

Focussing the attention on the applications to modern materials (laminates, sandwiches, foams among others) various improvements of the classical theory are suggested. For example, considering sandwich structures with soft core Reissner worked out a theory which takes into account the transverse shear which was ignored by Kirchhoff [24-26]. Similar governing equations (only some effects were not included) were derived by Mindlin introducing additional degrees of freedom for the points of the midplane [21]. On the classical approaches and their improvements, one can get more information, for example, from $[3,4,6,7,16,17]$.

Summarizing the monographs and reviews of the last 50 years (see $[5,11,13,19,22,27,28,33]$ ), one can conclude that there are two different possibilities to deduce the plate equations:

- Starting with the three-dimensional equations of solid mechanics the two-dimensional equations are established with the help of some hypotheses or mathematical treatment.

- Introducing a two-dimensional deformable surface the two-dimensional continuum equations are introduced by the direct approach.

The advantages/disadvantages are readily apparent. In the first case we get the constitutive equations by the same way like the other governing equations applying the hypotheses or the mathematical treatment of the, for example in the elastic case, Hooke's law. The correctness and the approximation degree are often unknown. The direct approach is in this sense better-the two-dimensional equations are physically-based and so exact like the three-dimensional continuum mechanics equations. But the constitutive equations must be identified which is a non-trivial problem. It was shown in $[2,5,32,33]$ among others that the concept of effective properties can support the handling of the identification. The goal of this paper is to show how a two-dimensional theory deduced with the help of the direct approach can be applied to plates made from FGM.

The discussion will be limited by the consideration of linear elastic material behavior and the geometrical linear relations. In contrast to the classical plate theory the deformations will be taken into account like in the theory of laminated plates with unsymmetrical cross-section properties [8]. The introduced theory is not restricted by the assumption that the transverse shear strains are neglected.

\section{Governing equations of a two-dimensional plate theory}

The direct approach is based on the Cosserat theory in continuum mechanics. In continuum mechanics any theory is based on the cutting principle, the assumption "actio = reactio" and some governing equations. Two different models can be introduced: the non-polar or the polar. In the first case only force actions are assumed. From this follows the symmetry of the stress tensor and only translations are considered. But in the basic course of mechanics the following basic equations are given: the static equilibrium for forces and moments or the dynamic equilibrium like the balance of momentum and the balance of moment of momentum (both are independent relations in the general case, which was shown, for example, by Truesdell [31]). For the second model one has force, and moments actions, too. From this it follows that there is a symmetric and a nonsymmetric stress tensor and translations and rotations can be introduced independently. Now any continuum (three-, two- or one-dimensional) can be introduced by a natural way: geometrical relations (kinematics), material independent balances of mass, momentum, moment of momentum, energy, entropy and the material dependent equations (constitutive equations and evolution equations). Finally, one needs boundary and, maybe, initial conditions. 


\subsection{Symbolic presentation of the equations}

Since the direct approach is a natural way to describe the behavior of plates (the stress resultants which are used in most of plate theories can be interpreted as forces and moments) a two-dimensional plate theory which allows one to model homogeneous and inhomogeneous plates can be presented as follows.

\subsubsection{Linear basic equations}

Let us introduce two basic assumptions:

Assumption 1 The plate (homogeneous or inhomogeneous in transverse direction) can be represented by a deformable surface.

Assumption 2 Each material point is an infinitesimal rigid body with five degrees of freedom (three translations and two rotations).

In addition, the theory presented here is limited by small displacements and rotations and the quadratic strain energy. The basics are presented in $[5,6,32]$. The following governing equations can be introduced:

- First and second Euler's law (balances of momentum and momentum of momentum)

$$
\nabla \cdot \boldsymbol{T}+q=\rho \ddot{\boldsymbol{u}}+\rho \Theta_{1} \cdot \ddot{\varphi}, \quad \nabla \cdot M+T_{\times}+\boldsymbol{m}=\rho \Theta_{1}^{\mathrm{T}} \cdot \ddot{\boldsymbol{u}}+\rho \Theta_{2} \cdot \ddot{\varphi}
$$

Here $\boldsymbol{T}, \boldsymbol{M}$ are the tensors of forces and moments, $\boldsymbol{q}, \boldsymbol{m}$ are the surface loads (forces and moments), $T_{\times}$is the vector invariant of the force tensor [20], $\nabla$ is the nabla (Hamilton) operator, $\boldsymbol{u}, \boldsymbol{\varphi}$ are the displacements and the rotations, $\Theta_{1}, \Theta_{2}$ are the first and the second tensor of inertia, $\rho$ is the density (effective property of the deformable surface $),(\ldots)^{\mathrm{T}}$ denotes the transposed tensor and $(. .$.$) the time derivative.$

- Geometrical relations

$$
\mu=(\nabla u \cdot a)^{\mathrm{sym}}, \quad \gamma=\nabla u \cdot n+c \cdot \varphi, \quad \kappa=\nabla \varphi
$$

$\boldsymbol{a}$ is the first metric tensor (plane tensor, that means two-dimensional!), $\boldsymbol{n}$ is the unit normal vector, $\boldsymbol{c}=$ $-a \times n$ is the discriminant tensor [33], $\mu, \gamma$ and $\kappa$ are deformation tensors (tensor of in-plane strains, vector of transverse shear strains and the tensor of the out-of-plane strains), $(. .)^{\mathrm{sym}}$ denotes symmetric part of the tensor.

- Boundary conditions

$$
\boldsymbol{v} \cdot \boldsymbol{T}=\boldsymbol{f}, \quad \boldsymbol{v} \cdot \boldsymbol{M}=\boldsymbol{l}(\boldsymbol{l} \cdot \boldsymbol{n}=0) \text { or } \boldsymbol{u}=\boldsymbol{u}^{0}, \quad \varphi=\varphi^{0} \quad \text { along } S
$$

Here $f$ and $\boldsymbol{l}$ are an external force and a couple acting along the boundary of plate $S$, while $\boldsymbol{u}^{0}$ and $\varphi^{0}$ are given functions describing the displacements and the rotation of the plate boundary, respectively. $\boldsymbol{v}$ is the unit normal vector to $S(v \cdot n=0)$. The relations (4) are the static and kinematic boundary conditions. Other types of boundary conditions are also possible. For example, the boundary conditions corresponding to a hinge are given by

$$
v \cdot M \cdot \tau=0, \quad u=0, \quad \varphi \cdot \tau=0
$$

Here $\tau$ is the unit tangent vector to $S(\tau \cdot n=\tau \cdot v=0)$.

\subsubsection{Two-dimensional constitutive equations}

Limiting our discussion to the elastic behavior and small strains (for example, rubber-like materials cannot be analyzed by these equations) the following statements for the constitutive modeling can be done. At first, the strain energy can be expended in a Taylor series limited by quadratic terms. In addition, we assume that the eigenstresses can be neglected (the linear terms in the series are dropped out). At second, the positive definiteness is guarantied.

- Strain energy of the deformable surface $W$

$$
W(\mu, \gamma, \kappa)=\frac{1}{2} \mu \cdot \cdot A \cdot \mu+\mu \cdot \boldsymbol{B} \cdot \kappa+\frac{1}{2} \kappa \cdot C \cdot \kappa+\frac{1}{2} \gamma \cdot \Gamma \cdot \gamma+\gamma \cdot\left(\Gamma_{1} \cdot \mu+\Gamma_{2} \cdot \kappa\right)
$$

$A, B, C$ are fourth rank tensors, $\Gamma_{1}, \Gamma_{2}$ are third rank tensors, $\Gamma$ is a second rank tensor of the effective stiffness properties. They depend on the material properties and the thickness geometry. In the general case the tensors contain 36 different values - a reduction is possible assuming some symmetries. 
- Constitutive equations

- Membrane forces

$$
T \cdot \boldsymbol{a}=\frac{\partial W}{\partial \boldsymbol{\mu}}=A \cdot \cdot \boldsymbol{\mu}+B \cdot \cdot \kappa+\gamma \cdot \Gamma_{1}
$$

- Transverse forces

$$
T \cdot \boldsymbol{n}=\frac{\partial W}{\partial \gamma}=\Gamma \cdot \boldsymbol{\gamma}+\Gamma_{1} \cdot \boldsymbol{\mu}+\Gamma_{2} \cdot \kappa
$$

- Moments

$$
\boldsymbol{M}^{\mathrm{T}}=\frac{\partial W}{\partial \boldsymbol{\kappa}}=\mu \cdot \cdot B+\boldsymbol{C} \cdot \boldsymbol{\kappa}+\boldsymbol{\gamma} \cdot \boldsymbol{\Gamma}_{2}
$$

\subsection{Basic equations in Cartesian coordinates}

Let us assume the Cartesian coordinate system $x_{1}, x_{2}$ (in-plane coordinates) and $z$ (orthogonal to the midplane). Then the unit normal vectors are $e_{1}, e_{2}$ and $n$. With respect to the introduced coordinate system the following representations are valid:

- Displacement and rotation vectors

$$
\boldsymbol{u}=u_{1} e_{1}+u_{2} \boldsymbol{e}_{2}+w \boldsymbol{n}, \quad \varphi=-\varphi_{2} e_{1}+\varphi_{1} \boldsymbol{e}_{2}
$$

$u_{\alpha}(\alpha=1,2)$ are the in-plane displacements, $w$ is the deflection and $\varphi_{\alpha}$ are the rotations about the axes $e_{\alpha}$, respectively.

- Force and moment tensors

$$
\begin{aligned}
& \boldsymbol{T}=T_{1} \boldsymbol{e}_{1} \boldsymbol{e}_{1}+T_{12}\left(\boldsymbol{e}_{1} \boldsymbol{e}_{2}+\boldsymbol{e}_{2} \boldsymbol{e}_{1}\right)+T_{2} \boldsymbol{e}_{2} \boldsymbol{e}_{2}+T_{1 n} \boldsymbol{e}_{1} \boldsymbol{n}+T_{2 n} \boldsymbol{e}_{2} \boldsymbol{n} \\
& \boldsymbol{M}=M_{1} \boldsymbol{e}_{1} \boldsymbol{e}_{2}-M_{12}\left(e_{1} e_{1}-e_{2} \boldsymbol{e}_{2}\right)-M_{2} e_{2} e_{1}
\end{aligned}
$$

$T_{\alpha}, T_{12}$ are the in-plane forces, $T_{\alpha n}$ are the transverse shear forces, $M_{\alpha}$ are the bending moments and $M_{12}$ is the torsion moment.

- Deformation tensors

$$
\begin{aligned}
& \boldsymbol{\mu}=\mu_{1} \boldsymbol{e}_{1} \boldsymbol{e}_{1}+\mu_{12}\left(\boldsymbol{e}_{1} \boldsymbol{e}_{2}+\boldsymbol{e}_{2} \boldsymbol{e}_{1}\right)+\mu_{2} \boldsymbol{e}_{2} \boldsymbol{e}_{2} \\
& \gamma=\gamma_{1} \boldsymbol{e}_{1}+\gamma_{2} \boldsymbol{e}_{2} \\
& \kappa=\kappa_{1} \boldsymbol{e}_{1} \boldsymbol{e}_{2}-\kappa_{12} \boldsymbol{e}_{1} \boldsymbol{e}_{1}+\kappa_{21} e_{2} \boldsymbol{e}_{2}-\kappa_{2} \boldsymbol{e}_{2} \boldsymbol{e}_{1}
\end{aligned}
$$

- External loads

$$
q=q_{1} e_{1}+q_{2} e_{2}+q_{n} n, \quad \boldsymbol{m}=-m_{2} e_{1}+m_{1} e_{2}
$$

How the symmetries of the "microstructure" do affect the physical properties? The answer comes from the Curie-Neumann's principle in the physics of crystals [23]:

Curie-Neumann's principle (first formulation): Any type of symmetry exhibited by the point group of a crystal is possessed by every physical property of the crystal.

Curie-Neumann's principle (second formulation): For a material element and for any of its physical properties, every material symmetry transformation of the material element is a physical symmetry transformation of the physical property.

Curie-Neumann's principle (third formulation): The symmetry group of the reason belongs to the symmetry group of the consequence.

The symmetry group of the "reasons" for the plate is the intersection of:

- Symmetry of the material of the plate (fibre-reinforced material, rolled sheets, ...),

- Symmetry of the surface shape (shell or plate) 
- Symmetry of the internal structure of the plate (laminated plates-symmetry of the stacking sequence with respect to the mid-surface, ...)

Let us consider an orthotropic material behavior and a plane mid-surface. In this case instead of the general form of the effective stiffness tensors, one gets [33]

$$
\begin{aligned}
& \boldsymbol{A}=A_{11} \boldsymbol{a}_{1} \boldsymbol{a}_{1}+A_{12}\left(a_{1} a_{2}+\boldsymbol{a}_{2} \boldsymbol{a}_{1}\right)+A_{22} a_{2} a_{2}+A_{44} a_{4} a_{4}, \\
& \boldsymbol{B}=B_{13} \boldsymbol{a}_{1} \boldsymbol{a}_{3}+B_{14} \boldsymbol{a}_{1} \boldsymbol{a}_{4}+B_{23} \boldsymbol{a}_{2} \boldsymbol{a}_{3}+\boldsymbol{B}_{24} \boldsymbol{a}_{2} \boldsymbol{a}_{4}+B_{42} \boldsymbol{a}_{4} \boldsymbol{a}_{2}, \\
& \boldsymbol{C}=C_{22} a_{2} a_{2}+C_{33} \boldsymbol{a}_{3} \boldsymbol{a}_{3}+C_{34}\left(a_{3} a_{4}+a_{4} a_{3}\right)+C_{44} \boldsymbol{a}_{4} \boldsymbol{a}_{4}, \\
& \boldsymbol{\Gamma}=\Gamma_{1} \boldsymbol{a}_{1}+\Gamma_{2} \boldsymbol{a}_{2}, \quad \boldsymbol{\Gamma}_{1}=0, \quad \Gamma_{2}=0
\end{aligned}
$$

with

$$
a_{1}=a=e_{1} e_{1}+e_{2} e_{2}, \quad a_{2}=e_{1} e_{1}-e_{2} e_{2}, \quad a_{3}=c=e_{1} e_{2}-e_{2} e_{1}, \quad a_{4}=e_{1} e_{2}+e_{2} e_{1}
$$

$e_{1}, e_{2}$ are unit basic vectors. In addition, one obtains the orthogonality condition for $a_{i}(i=1,2,3,4)$

$$
\frac{1}{2} \boldsymbol{a}_{i} \cdot \boldsymbol{a}_{j}=\delta_{i j}, \quad \delta_{i j}=\left\{\begin{array}{l}
1, i=j, \\
0, i \neq j
\end{array}\right.
$$

Now the first and the second Euler's law, the geometrical relations, and the constitutive equations take the form (static case):

- First and second Euler's law

$$
\begin{array}{ll}
T_{1,1}+T_{12,2}+q_{1}=0, & T_{12,1}+T_{2,2}+q_{2}=0, \\
T_{1 n, 1}+T_{2 n, 2}+q_{n}=0, & \\
M_{1,1}+M_{12,2}-T_{1 n}+m_{1}=0, & M_{12,1}+M_{2,2}-T_{2 n}+m_{2}=0
\end{array}
$$

- Boundary conditions (for brevity, we present it when $S$ is a part of line $x_{1}=$ const $\left(\boldsymbol{v}=\boldsymbol{e}_{1}, \boldsymbol{\tau}=\boldsymbol{e}_{2}\right)$ ) Static boundary conditions

$$
T_{1}=f_{1}, \quad T_{12}=f_{2}, \quad T_{1 n}=f_{n}, \quad M_{1}=l_{1}, \quad M_{12}=l_{2}
$$

Kinematic boundary conditions

$$
u_{1}=u_{1}^{0}, \quad u_{2}=u_{2}^{0}, \quad w=w^{0}, \quad \varphi_{1}=\varphi_{1}^{0}, \quad \varphi_{2}=\varphi_{2}^{0},
$$

Boundary conditions for a hinge

$$
M_{1}=0, \quad u_{1}=u_{2}=w=0, \quad \varphi_{2}=0
$$

- Geometrical relations

$$
\begin{aligned}
& \mu_{1}=u_{1,1}, \quad \mu_{2}=u_{2,2}, \quad \mu_{12}=\frac{1}{2}\left(u_{1,2}+u_{2,1}\right), \\
& \gamma_{1}=w_{, 1}+\varphi_{1}, \gamma_{2}=w_{, 2}+\varphi_{2} \\
& \kappa_{1}=\varphi_{1,1}, \quad \kappa_{2}=\varphi_{2,2}, \quad \kappa_{12}=\varphi_{2,1}, \quad \kappa_{21}=\varphi_{1,2}
\end{aligned}
$$

- Constitutive equations

$$
\begin{aligned}
T_{1}= & \left(A_{11}+2 A_{12}+A_{22}\right) \mu_{1}+\left(A_{11}-A_{22}\right) \mu_{2}-\left(B_{13}+B_{23}-B_{14}-B_{24}\right) \kappa_{1} \\
& -\left(B_{13}+B_{23}+B_{14}+B_{24}\right) \kappa_{2}, \\
T_{2}= & \left(A_{11}-A_{22}\right) \mu_{1}+\left(A_{11}-2 A_{12}+A_{22}\right) \mu_{2}-\left(B_{13}-B_{23}-B_{14}+B_{24}\right) \kappa_{1} \\
& -\left(B_{13}-B_{23}+B_{14}-B_{24}\right) \kappa_{2}, \\
T_{12}= & 2 \mu_{12} A_{44}+\left(\kappa_{12}+\kappa_{21}\right) B_{42}, \\
T_{1 n}= & \left(\Gamma_{1}+\Gamma_{2}\right) \gamma_{1}, \quad T_{2 n}=\left(\Gamma_{1}-\Gamma_{2}\right) \gamma_{2}, \\
M_{1}= & \left(-B_{13}-B_{23}+B_{14}+B_{24}\right) \mu_{1}-\left(B_{13}-B_{23}-B_{14}+B_{24}\right) \mu_{2} \\
& +\left(C_{33}-2 C_{34}+C_{44}\right) \kappa_{1}+\left(C_{33}-C_{44}\right) \kappa_{2}, \\
M_{2}= & -\left(B_{13}+B_{23}+B_{14}+B_{24}\right) \mu_{1}-\left(B_{13}-B_{23}+B_{14}-B_{24}\right) \mu_{2} \\
& +\left(C_{33}-C_{44}\right) \kappa_{1}+\left(C_{33}+2 C_{34}+C_{44}\right) \kappa_{2}, \\
M_{12}= & 2 \mu_{12} B_{42}+C_{22}\left(\kappa_{12}+\kappa_{21}\right)
\end{aligned}
$$

From the last equations it can be seen that the $A_{i j}$ are the effective in-plane stiffness tensor components, the $C_{i j}$ are the effective plate stiffness tensor components, $\Gamma_{\alpha}$ are the components of the transverse shear stiffness tensor and $B_{i j}$ are the components of the coupling stiffness tensor. 
The individuality of each class of plates in the framework of the direct approach is expressed by the effective properties (stiffness, density, inertia terms, etc). Let us focus our attention on the stiffness expressions. The identification of the effective stresses should be performed on the base of the properties of the real material. Let us assume Hooke's Law as

$$
\begin{aligned}
& \varepsilon_{1}=u_{1,1}^{*}=\frac{1}{E_{1}} \sigma_{1}-\frac{\nu_{21}}{E_{2}} \sigma_{2}-\frac{\nu_{n 1}}{E_{n}} \sigma_{n}, \\
& \varepsilon_{2}=u_{2,2}^{*}=\frac{1}{E_{2}} \sigma_{2}-\frac{\nu_{12}}{E_{1}} \sigma_{1}-\frac{v_{n 2}}{E_{n}} \sigma_{n}, \\
& \varepsilon_{n}=u_{n, n}^{*}=\frac{1}{E_{n}} \sigma_{n}-\frac{\nu_{1 n}}{E_{1}} \sigma_{1}-\frac{\nu_{2 n}}{E_{2}} \sigma_{2}, \\
& \gamma_{12}=u_{1,2}^{*}+u_{2,1}^{*}=\frac{\tau_{12}}{G_{12}}, \\
& \gamma_{n 1}=u_{1, n}^{*}+u_{n, 1}^{*}=\frac{\tau_{n 1}}{G_{n 1}}, \\
& \gamma_{2 n}=u_{2, n}^{*}+u_{n, 2}^{*}=\frac{\tau_{2 n}}{G_{2 n}}
\end{aligned}
$$

with $v_{i j} E_{j}=v_{j i} E_{i}$. Equations (18) are valid in the following cases:

Case 1 Homogeneous plates-all properties are constant (no dependency from $z$ ).

Case 2 Inhomogeneous plates (sandwich, multilayered, functionally graded) —all properties are functions of $z$, e.g., $E_{i}=E_{i}(z)$.

The identification of the effective properties can be performed with the help of static boundary value problems (two-dimensional, three-dimensional) and the comparison of the forces and moments (in the sense of averaged stresses or stress resultants)

$$
T=\langle\boldsymbol{a} \cdot \boldsymbol{\sigma}\rangle, \quad \boldsymbol{M}=\langle\boldsymbol{a} \cdot \sigma z \cdot c\rangle
$$

$\sigma$ is the stress tensor, $\langle(\ldots)\rangle=\int_{-h / 2}^{h / 2}(\ldots) \mathrm{d} z$.

\subsubsection{Classical stiffness values}

Problem 1 Tension and bending The following two-dimensional kinematical field is given

$$
u=D_{1} x_{1} e_{1}+D_{2} x_{2} e_{2}-\frac{1}{2}\left(\frac{x_{1}^{2}}{R_{1}}+\frac{x_{2}^{2}}{R_{2}}\right) n, \quad \varphi=-\frac{x_{2}}{R_{2}} e_{1}+\frac{x_{1}}{R_{1}} e_{2}
$$

$D_{1}, D_{2}, R_{1}$ and $R_{2}$ are constants. Two-dimensional strain tensors can be calculated

$$
\boldsymbol{\mu}=D_{1} \boldsymbol{e}_{1} \boldsymbol{e}_{1}+D_{2} \boldsymbol{e}_{2} \boldsymbol{e}_{2}, \quad \gamma=0, \quad \kappa=\frac{1}{R_{1}} \boldsymbol{e}_{1} \boldsymbol{e}_{2}-\frac{1}{R_{2}} \boldsymbol{e}_{2} \boldsymbol{e}_{1}
$$


and one gets the two-dimensional constitutive equations

$$
\begin{aligned}
T_{1}= & D_{1}\left(A_{11}+2 A_{12}+A_{22}\right)+D_{2}\left(A_{11}-A_{22}\right)-\frac{1}{R_{1}}\left(B_{13}+B_{23}-B_{14}-B_{24}\right) \\
& -\frac{1}{R_{2}}\left(B_{13}+B_{23}+B_{14}+B_{24}\right) \\
T_{2}= & D_{1}\left(A_{11}-A_{22}\right)+D_{2}\left(A_{11}-2 A_{12}+A_{22}\right)-\frac{1}{R_{1}}\left(B_{13}-B_{23}-B_{14}+B_{24}\right) \\
& -\frac{1}{R_{2}}\left(B_{13}-B_{23}+B_{14}-B_{24}\right) \\
M_{1}= & D_{1}\left(-B_{13}-B_{23}+B_{14}+B_{24}\right)-D_{2}\left(B_{13}-B_{23}-B_{14}+B_{24}\right) \\
& +\frac{1}{R_{1}}\left(C_{33}-2 C_{34}+C_{44}\right)+\frac{1}{R_{2}}\left(C_{33}-C_{44}\right) \\
M_{2}= & -D_{1}\left(B_{13}+B_{23}+B_{14}+B_{24}\right)-D_{2}\left(B_{13}-B_{23}+B_{14}-B_{24}\right) \\
& +\frac{1}{R_{1}}\left(C_{33}-C_{44}\right)+\frac{1}{R_{2}}\left(C_{33}+2 C_{34}+C_{44}\right)
\end{aligned}
$$

Now assuming the three-dimensional strain tensor components corresponding to the two-dimensional problem

$$
\varepsilon_{1}=D_{1}+\frac{z}{R_{1}}, \quad \varepsilon_{2}=D_{2}+\frac{z}{R_{2}}
$$

the stress tensor components under assumptions of the plane stress state and the Hooke's law (18) are

$$
\sigma_{1}=\frac{E_{1}}{1-v_{12} \nu_{21}}\left(\varepsilon_{1}+v_{21} \varepsilon_{2}\right), \quad \sigma_{2}=\frac{E_{2}}{1-v_{12} \nu_{21}}\left(\varepsilon_{2}+v_{12} \varepsilon_{1}\right)
$$

Finally, the stress resultants can be estimated

$$
\begin{aligned}
& T_{1}=D_{1}\left\langle\frac{E_{1}}{1-v_{12} v_{21}}\right\rangle+\frac{1}{R_{1}}\left\langle\frac{E_{1} z}{1-v_{12} v_{21}}\right\rangle+D_{2}\left\langle\frac{v_{21} E_{1}}{1-v_{12} v_{21}}\right\rangle+\frac{1}{R_{2}}\left\langle\frac{v_{21} E_{1} z}{1-v_{12} v_{21}}\right\rangle, \\
& T_{2}=D_{2}\left\langle\frac{E_{2}}{1-v_{12} v_{21}}\right\rangle+\frac{1}{R_{2}}\left\langle\frac{E_{2} z}{1-v_{12} v_{21}}\right\rangle+D_{1}\left\langle\frac{v_{12} E_{2}}{1-v_{12} v_{21}}\right\rangle+\frac{1}{R_{1}}\left\langle\frac{v_{12} E_{2} z}{1-v_{12} v_{21}}\right\rangle, \\
& M_{1}=D_{1}\left\langle\frac{E_{1} z}{1-v_{12} v_{21}}\right\rangle+\frac{1}{R_{1}}\left\langle\frac{E_{1} z^{2}}{1-v_{12} v_{21}}\right\rangle+D_{2}\left\langle\frac{v_{21} E_{1} z}{1-v_{12} v_{21}}\right\rangle+\frac{1}{R_{2}}\left\langle\frac{v_{21} E_{1} z^{2}}{1-v_{12} v_{21}}\right\rangle, \\
& M_{2}=D_{2}\left\langle\frac{E_{2} z}{1-v_{12} v_{21}}\right\rangle+\frac{1}{R_{2}}\left\langle\frac{E_{2} z^{2}}{1-v_{12} v_{21}}\right\rangle+D_{1}\left\langle\frac{v_{12} E_{2} z}{1-v_{12} v_{21}}\right\rangle+\frac{1}{R_{1}}\left\langle\frac{v_{12} E_{2} z^{2}}{1-v_{12} v_{21}}\right\rangle
\end{aligned}
$$

The comparison of the stress resultants (19) and (20) allows the calculation of the following stiffness tensor components

$$
\begin{aligned}
& A_{11}=\frac{1}{4}\left\langle\frac{E_{1}+E_{2}+2 E_{1} v_{21}}{1-v_{12} v_{21}}\right\rangle, \quad A_{12}=\frac{1}{4}\left\langle\frac{E_{1}-E_{2}}{1-v_{12} v_{21}}\right\rangle, \\
& A_{22}=\frac{1}{4}\left\langle\frac{E_{1}+E_{2}-2 E_{1} v_{21}}{1-v_{12} v_{21}}\right\rangle \\
& B_{13}=-\frac{1}{4}\left\langle\frac{E_{1}+E_{2}+2 E_{1} v_{21}}{1-v_{12} v_{21}} z\right\rangle,-B_{23}=B_{14}=\frac{1}{4}\left\langle\frac{E_{1}-E_{2}}{1-v_{12} v_{21}} z\right\rangle, B_{24}=\frac{1}{4}\left\langle\frac{E_{1}+E_{2}-2 E_{1} v_{21}}{1-v_{12} v_{21}} z\right\rangle \text {, } \\
& C_{33}=\frac{1}{4}\left\langle\frac{E_{1}+E_{2}+2 E_{1} v_{21}}{1-v_{12} v_{21}} z^{2}\right\rangle, \quad C_{34}=-\frac{1}{4}\left\langle\frac{E_{1}-E_{2}}{1-v_{12} v_{21}} z^{2}\right\rangle, \quad C_{44}=\frac{1}{4}\left\langle\frac{E_{1}+E_{2}-2 E_{1} v_{21}}{1-v_{12} v_{21}} z^{2}\right\rangle
\end{aligned}
$$

Problem 2 Plane shear Let us assume the two-dimensional kinematical field

$$
u=S_{1} x_{2} \boldsymbol{e}_{1}+S_{1} x_{1} e_{2}-S_{2} x_{1} x_{2} n, \quad \varphi=-S_{2}\left(x_{1} \boldsymbol{e}_{1}-x_{2} \boldsymbol{e}_{2}\right)
$$

The corresponding three-dimensional strain tensor component is

$$
\gamma_{12}=u_{1,2}^{*}+u_{2,1}^{*}=S_{1}+S_{2} z
$$

The comparison results in

$$
A_{44}=\left\langle G_{12}\right\rangle, \quad B_{42}=-\left\langle G_{12} z\right\rangle, \quad C_{22}=\left\langle G_{12} z^{2}\right\rangle
$$


Problem 3 Torsion Let us introduce a deformable strip $\left(\left|x_{1}\right| \leq l_{1},\left|x_{2}\right|<\infty\right)$ under constant torsion moment at the boundaries $x_{1}= \pm l_{1}$. Then one gets the two-dimensional kinematical field

$$
u=u_{2}\left(x_{1}\right) e_{2}, \quad \varphi=-\varphi_{1}\left(x_{1}\right) e_{1}
$$

and the force and moment tensors

$$
T=\left(A_{44} u_{2,1}-B_{42} \varphi_{2,1}\right) a_{4}+\left(\Gamma_{1}-\Gamma_{2}\right) \varphi_{2} e_{2} \boldsymbol{n}, \quad \boldsymbol{M}=\left(B_{42} u_{2,1}-C_{22} \varphi_{2,1}\right) a_{2}
$$

The dual three-dimensional problem (strip $\left|x_{1}\right| \leq l_{1},\left|x_{2}\right|<\infty,|z| \leq h / 2$ ) results in the displacements

$$
u_{1}^{*}=w^{*}=0, \quad u_{2}^{*}=u_{2}^{*}\left(x_{1}, z\right)
$$

the stress tensor

$$
\boldsymbol{\sigma}=G_{12} \frac{\partial u_{2}^{*}}{\partial x_{1}} a_{4}+G_{2 n} \frac{\partial u_{2}^{*}}{\partial z}\left(e_{2} \boldsymbol{n}+\boldsymbol{n} e_{2}\right)
$$

and equilibrium equation

$$
G_{12} \frac{\partial^{2} u_{2}^{*}}{\partial x_{1}^{2}}+\frac{\partial}{\partial z}\left(G_{2 n} \frac{\partial u_{2}^{*}}{\partial z}\right)=0
$$

The solution with respect to the boundary conditions $\sigma_{n}=\tau_{1 n}=\tau_{2 n}=0$ at $|z|=h / 2$ can be obtained by the following Fourier's ansatz: $u_{2}^{*}\left(x_{1}, z\right)=X\left(x_{1}\right) Z(z)$, which yields a Sturm-Liouville problem

$$
\frac{\mathrm{d}}{\mathrm{d} z}\left(G_{2 n} \frac{\mathrm{d} Z}{\mathrm{~d} z}\right)+\lambda_{*}^{2} G_{12} Z=0,\left.\quad \frac{\mathrm{d} Z}{\mathrm{~d} z}\right|_{|z|=\frac{h}{2}}=0
$$

and

$$
\frac{\mathrm{d}^{2} X}{\mathrm{~d} x_{1}^{2}}-\lambda_{*}^{2} X=0
$$

The lowest non-trivial positive solution $\lambda_{*}$ one obtains from

$$
X\left(x_{1}\right)=B \frac{\sinh \lambda_{*} x_{1}}{\lambda_{*} \cosh \lambda_{*} l_{1}} \text { and } u_{2}^{*}=B Z(z) \frac{\sinh \lambda_{*} x_{1}}{\lambda_{*} \cosh \lambda_{*} l_{1}}
$$

Finally, after comparison of the two-dimensional and the three-dimensional solutions one gets

$$
\lambda=\lambda_{*}=\sqrt{\frac{\left(\Gamma_{1}-\Gamma_{2}\right) A_{44}}{A_{44} C_{22}-B_{42}^{2}}}
$$

$T_{12}$ and $M_{12}$ obtained by the two-dimensional and the three-dimensional approaches are in a full agreement. For the kinematical fields, one gets

$$
\begin{aligned}
\left\langle G_{12}\left(u_{2}^{*}-u_{2}-z \varphi_{2}\right)^{2}\right\rangle & =\min \left(u_{2}, \varphi_{2}\right) \\
u_{2} & =\frac{M_{12}^{*}\left\langle G_{12} z\right\rangle}{\left\langle G_{12}\right\rangle\left\langle G_{12} z^{2}\right\rangle-\left\langle G_{12} z^{2}\right\rangle} \frac{\sinh \lambda_{*} x_{1}}{\lambda_{*} \cosh \lambda_{*} l_{1}}, \\
\varphi_{2} & =-\frac{M_{12}^{*}\left\langle G_{12}\right\rangle}{\left\langle G_{12}\right\rangle\left\langle G_{12} z^{2}\right\rangle-\left\langle G_{12} z^{2}\right\rangle} \frac{\sinh \lambda_{*} x_{1}}{\lambda_{*} \cosh \lambda_{*} l_{1}}
\end{aligned}
$$

In addition, one has to analyze the similar problem for the second direction in the two-dimensional case: $\left(\left|x_{1}\right|<\infty,\left|x_{2}\right| \leq l_{2}\right)$ as in the three-dimensional case. $\left(\left|x_{1}\right|<\infty,\left|x_{2}\right| \leq l_{2},|z| \leq h / 2\right)$ with the constant torsion moment at the boundary $\left|x_{2}\right| \leq l_{2}$. Now the one-dimensional case results in

$$
\frac{\mathrm{d}}{\mathrm{d} z}\left(G_{1 n} \frac{\mathrm{d} \tilde{Z}}{\mathrm{~d} z}\right)+\eta^{2} G_{12} \tilde{Z}=0,\left.\quad \frac{\mathrm{d} \tilde{Z}}{\mathrm{~d} z}\right|_{|z|=\frac{h}{2}}=0, \quad \eta=\sqrt{\frac{\left(\Gamma_{1}+\Gamma_{2}\right) A_{44}}{A_{44} C_{22}-B_{42}^{2}}}
$$

Finally, we get the following expressions for the transverse shear stiffness tensor components

$$
\Gamma_{1}=\frac{1}{2}\left(\lambda^{2}+\eta^{2}\right) \frac{A_{44} C_{22}-B_{42}^{2}}{A_{44}}, \quad \Gamma_{2}=\frac{1}{2}\left(\eta^{2}-\lambda^{2}\right) \frac{A_{44} C_{22}-B_{42}^{2}}{A_{44}}
$$


2.4 Special case-isotropic behavior

In the case of isotropic material behavior, one has to set in Eq. (18)

$$
E_{1}=E_{2}=E_{n}=E(z), \quad v_{i j}=v(z), \quad G_{12}=G_{n 1}=G_{2 n}=G(z)=\frac{E(z)}{2(1+v(z))}
$$

Instead of (21-23), the following non-zero components of the stiffness tensors are valid in-plane stiffness tensor components

$$
A_{11}=\frac{1}{2}\left\langle\frac{E}{1-v}\right\rangle, \quad A_{22}=\frac{1}{2}\left\langle\frac{E}{1+v}\right\rangle=A_{44}=\langle G\rangle,
$$

coupling stiffness tensor components

$$
B_{13}=-\frac{1}{2}\left\langle\frac{E}{1-v} z\right\rangle, \quad B_{24}=\frac{1}{2}\left\langle\frac{E}{1+v} z\right\rangle=-B_{42}=\langle G z\rangle,
$$

plate stiffness tensor components

$$
C_{33}=\frac{1}{2}\left\langle\frac{E}{1-v} z^{2}\right\rangle, \quad C_{44}=\frac{1}{2}\left\langle\frac{E}{1+v} z^{2}\right\rangle=C_{22}=\left\langle G z^{2}\right\rangle,
$$

transverse shear stiffness tensor components

$$
\Gamma_{1}=\lambda^{2} \frac{A_{44} C_{22}-B_{42}^{2}}{A_{44}}
$$

with $\lambda$ following from

$$
\frac{\mathrm{d}}{\mathrm{d} z}\left(G \frac{\mathrm{d} Z}{\mathrm{~d} z}\right)+\lambda_{*}^{2} G Z=0,\left.\quad \frac{\mathrm{d} Z}{\mathrm{~d} z}\right|_{|z|=\frac{h}{2}}=0
$$

\section{Examples of effective stiffness properties}

\subsection{Homogeneous plate}

The simplest test for the correctness of the estimated stiffness properties is the homogeneous isotropic plate. The basic geometrical property is the thickness $h$, the plate is symmetrical with respect to the mid-plane which results in $\boldsymbol{B} \equiv \mathbf{0}$. Let us assume the following material data: the Young's modulus $E$ and the shear modulus $G=E / 2(1+v), v$ is the Poisson's ratio. All material properties are constant, that means they do not depend on the thickness coordinate. The non-zero components of the classical stiffness tensors are

$$
\begin{aligned}
& A_{11}=\frac{E h}{2(1-v)}, \quad A_{22}=\frac{E h}{2(1+v)}=A_{44}=G h, \\
& C_{33}=\frac{E h^{3}}{24(1-v)}, C_{44}=\frac{E h^{3}}{24(1+v)}=C_{22}=\frac{G h^{3}}{12}
\end{aligned}
$$

The classical plate (bending) stiffness follows as

$$
C_{33}+C_{44}=\frac{E h^{3}}{12\left(1-v^{2}\right)}
$$

and can be found in the classical textbooks, e.g., [30]. The transverse shear stiffness follows from

$$
\Gamma=\lambda^{2} C_{22}
$$

with

$$
\frac{\mathrm{d}^{2} Z}{\mathrm{~d} z^{2}}+\lambda^{2} Z=0,\left.\quad \frac{\mathrm{d} Z}{\mathrm{~d} z}\right|_{|z|=\frac{h}{2}}=0
$$


The solution $\cos \lambda z=0$ yields the smallest eigenvalue $\lambda=\frac{\pi}{h}$, and finally one obtains

$$
\Gamma=\frac{\pi^{2}}{h^{2}} \frac{G h^{3}}{12}=\frac{\pi^{2}}{12} G h
$$

$\pi^{2} / 12$ is a factor which plays in other theories the role of the shear correction. Comparing this value with the Mindlin's estimate $\pi^{2} / 12$ [21] and Reissner's estimate 5/6 [24] one concludes that the direct approach yields the same value like in the Mindlin's theory (note that Mindlin's shear correction is based on the solution of a dynamical problem, here the solution of a statical problem) was used. The Reissner's value slightly differs.

\subsection{Classical sandwich plate in Reissner's sense}

Now we assume the following geometry: $h_{\mathrm{c}}$ is the core thickness and $h_{\mathrm{f}}$ the thickness of the face sheets $\left(h_{\mathrm{c}} \gg h_{\mathrm{f}}\right)$. The material properties of the core and the face sheets are $E_{\mathrm{c}}, E_{\mathrm{f}}, G_{\mathrm{c}}, G_{\mathrm{f}}$ with $E_{\mathrm{c}} \ll E_{\mathrm{f}}, G_{\mathrm{c}} \ll G_{\mathrm{f}}$. We have again a symmetry with respect to the mid-plane that means $\boldsymbol{B} \equiv \mathbf{0}$. With the thickness $h=h_{\mathrm{c}}+h_{\mathrm{f}}$ one gets

$$
\begin{aligned}
& A_{11}=\frac{1}{2}\left(\frac{E_{\mathrm{f}} h_{\mathrm{f}}}{1-v_{\mathrm{f}}}+\frac{E_{\mathrm{c}} h_{\mathrm{c}}}{1-v_{\mathrm{c}}}\right), \quad A_{22}=\frac{1}{2}\left(\frac{E_{\mathrm{f}} h_{\mathrm{f}}}{1+v_{\mathrm{f}}}+\frac{E_{\mathrm{c}} h_{\mathrm{c}}}{1+v_{\mathrm{c}}}\right)=A_{44}, \\
& C_{33}=\frac{1}{24}\left[\frac{E_{\mathrm{f}}\left(h^{3}-h_{\mathrm{c}}^{3}\right)}{1-v_{\mathrm{f}}}+\frac{E_{\mathrm{c}} h_{\mathrm{c}}^{3}}{1-v_{\mathrm{c}}}\right], C_{44}=\frac{1}{24}\left[\frac{E_{\mathrm{f}}\left(h^{3}-h_{\mathrm{c}}^{3}\right)}{1+v_{\mathrm{f}}}+\frac{E_{\mathrm{c}} h_{\mathrm{c}}}{1+v_{\mathrm{c}}}\right]=C_{22}
\end{aligned}
$$

The bending stiffness results in

$$
C_{33}+C_{44}=\frac{1}{12}\left[\frac{E_{\mathrm{f}}\left(h^{3}-h_{\mathrm{c}}^{3}\right)}{1-v_{\mathrm{f}}^{2}}+\frac{E_{\mathrm{c}} h_{\mathrm{c}}^{3}}{1-v_{\mathrm{c}}^{2}}\right]
$$

The transverse shear stiffness can be computed by

$$
Z(z)=\left\{\begin{array}{cc}
A \cos \lambda\left(z-\frac{h}{2}\right) & \frac{h_{\mathrm{c}}}{2} \leq z \leq \frac{h}{2}, \\
B \sin \lambda z & |z| \leq \frac{h_{\mathrm{c}}}{2} \\
-A \cos \lambda\left(z+\frac{h}{2}\right) & -\frac{h}{2} \leq z \leq-\frac{h_{\mathrm{c}}}{2}
\end{array}\right.
$$

This results in a transcendent equation

$$
\mu \cos \lambda \frac{h_{\mathrm{f}}}{2} \cos \lambda \frac{h_{\mathrm{c}}}{2}-\sin \lambda \frac{h_{\mathrm{f}}}{2} \sin \lambda \frac{h_{\mathrm{c}}}{2}=0
$$

or

$$
\mu \cos \gamma(1-\alpha) \cos \gamma \alpha-\sin \gamma(1-\alpha) \sin \gamma \alpha=0
$$

with

$$
\gamma=\lambda \frac{h}{2}, \quad \alpha=\frac{h_{\mathrm{c}}}{h}
$$

$\gamma$ and $\alpha$ take the values $0 \leq \mu=G_{\mathrm{c}} / G_{\mathrm{f}}<\infty$ and $0 \leq \alpha \leq 1$. A typical sandwich structure has a very weak core. Then the bending stiffness can be approximated by

$$
C_{33}+C_{44}=\frac{1}{4} \frac{E_{\mathrm{f}} h^{2} h_{\mathrm{f}}}{1-v_{\mathrm{f}}^{2}},
$$

the transverse shear stiffness by $\Gamma=G_{\mathrm{c}} h$. This solution was first obtained by Reissner [26]. 


\subsection{Functionally graded material}

Functionally graded materials (FGM) are composite materials where the composition or the microstructure are locally varied so that a certain variation of the local material properties is achieved. A nitrided steel, for instance, could be also regarded as a FGM. Modern FGMs are constructed for complex requirements, such as the heat shield of a rocket or implants for humans. The gradual transition between the heat or corrosion resistant outer layer (often made of a ceramic material) and the tough metallic base material increases in most cases the life time of the component.

An example of FGM material is a porous material with nonhomogeneous distribution of porosity. Engineering structures made of porous materials, especially metal foams, have been used different applications in the last decades $[9,10,12]$. A metal foam is a cellular structure consisting of a solid metal, for example aluminium, steel, copper, etc., containing a large volume fraction of gas-filled pores. There are two types of metal foams. One is the closed-cell foam, while the second one is the open-cell foam. The defining characteristic of metal foams is a very high porosity: typically well over 80,90 and even $98 \%$ of the volume consists of void spaces. Another example of perspective material is a syntactic foam. They are composite materials synthesized by filling a metal, polymer or ceramic matrix with hollow particles called microballoons (see, e.g., [14, 15]).

\subsubsection{Linear symmetric distribution}

Now we have a functionally graded material with changing mechanical properties. The material is assumed to be isotropic. The mechanical properties are changing linearly and the changes are symmetrical with respect to the mid-plane. In addition, it will be assumed that the changing properties are related to the Young's and the shear modulus. To make the calculation as simple as possible the Poisson ratio is assumed to be approximately constant $(v \approx$ const). Then the distribution of the Young's and the shear modulus can be given as

$$
E(z)=E_{\mathrm{o}}\left[\frac{2}{h}|z|+\frac{E_{\mathrm{m}}}{E_{\mathrm{o}}}\left(1-\frac{2}{h}|z|\right)\right], \quad G(z)=G_{\mathrm{o}}\left[\frac{2}{h}|z|+\frac{G_{\mathrm{m}}}{G_{\mathrm{o}}}\left(1-\frac{2}{h}|z|\right)\right]
$$

Here the index o denotes the value on the outer surfaces $(z= \pm h / 2)$ and $\mathrm{m}$ at the mid-plane $(z=0)$. Due to the symmetry to the mid-plane $B \equiv 0$. The classical stiffness tensor components can be computed analytically

$$
\begin{aligned}
& A_{11}=\frac{E_{\mathrm{o}} h}{4(1-v)}\left(1+\frac{E_{\mathrm{m}}}{E_{\mathrm{o}}}\right), \quad A_{22}=\frac{E_{\mathrm{o}} h}{4(1+v)}\left(1+\frac{E_{\mathrm{m}}}{E_{\mathrm{o}}}\right)=A_{44}, \\
& C_{33}=\frac{E_{\mathrm{o}} h^{3}}{96(1-v)}\left(3+\frac{E_{\mathrm{m}}}{E_{\mathrm{o}}}\right), C_{44}=\frac{E_{\mathrm{o}} h^{3}}{96(1+v)}\left(3+\frac{E_{\mathrm{m}}}{E_{\mathrm{o}}}\right)=C_{22}
\end{aligned}
$$

The transverse shear stiffness tensor components results from

$$
\Gamma_{1}=\lambda^{2} C_{22}=\lambda^{2} \frac{G_{\mathrm{o}} h^{3}}{48}\left(3+\frac{G_{\mathrm{m}}}{G_{\mathrm{o}}}\right)
$$

with $\lambda$ following from

$$
\frac{\mathrm{d}}{\mathrm{d} z}\left[G(z) \frac{\mathrm{d} Z}{\mathrm{~d} z}\right]+\lambda^{2} G(z) Z=0,\left.\quad \frac{\mathrm{d} Z}{\mathrm{~d} z}\right|_{|z|=\frac{h}{2}}=0
$$

Instead of $G(z)$ one has to take the second equation of (29). This spectral problem was solved numerically by using the shooting method [29]. The dependence normalized $\Gamma_{1}$ versus $g_{\mathrm{m}} \equiv \frac{G_{\mathrm{m}}}{G_{\mathrm{o}}}$ is shown on the Fig. 1 (solid line), the Reissner's solution is presented by the dashed line.

Reissner's solution gives understated values of the transverse stiffness when the difference between elastic moduli is big enough. On the other hand, Reissner's solution gives overstated values when the elastic moduli do not differ. Reissner's formula gives us good coincidence with our results when the $g_{\mathrm{m}} \sim 0.6$. 


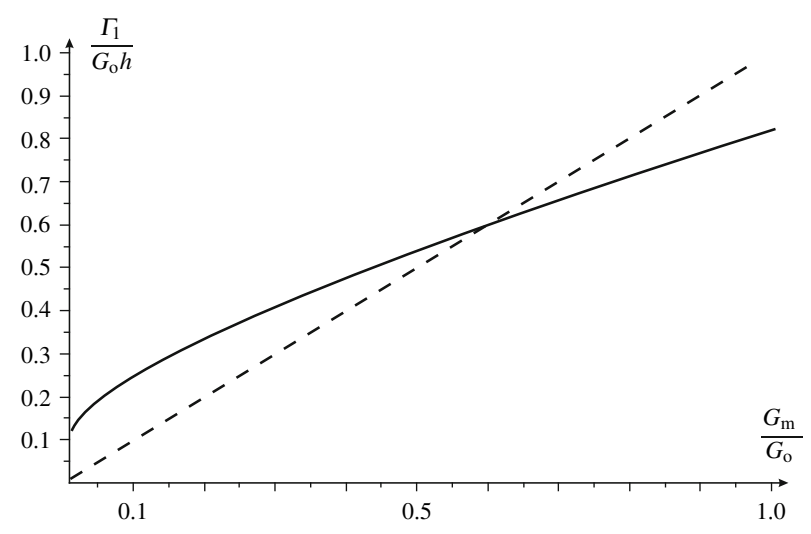

Fig. 1 Normalised transverse shear stiffness in dependence of the shear modulus

\subsubsection{Linear nonsymmetric distribution}

If $\mathrm{u}$ and 1 denote the values of the Young's modulus and the shear modulus on the upper surface $(z=h / 2)$ and the lower surface $(z=-h / 2)$, one can represent the linear distribution by

$$
E(z)=\frac{1}{2}\left(E_{\mathrm{u}}-E_{1}\right) z \frac{2}{h}+\frac{1}{2}\left(E_{\mathrm{u}}+E_{1}\right), \quad G(z)=\frac{1}{2}\left(G_{\mathrm{u}}-G_{1}\right) z \frac{2}{h}+\frac{1}{2}\left(G_{\mathrm{u}}+G_{1}\right)
$$

and with the assumption of the constant Poisson's ratio, one gets

$$
\begin{aligned}
& A_{11}=\frac{\left(E_{\mathrm{u}}+E_{1}\right) h}{4(1-v)}, \quad A_{22}=\frac{\left(E_{\mathrm{u}}+E_{1}\right) h}{4(1+v)}=A_{44}, \\
& B_{13}=-\frac{\left(E_{\mathrm{u}}-E_{1}\right) h^{2}}{24(1-v)}, B_{24}=\frac{\left(E_{\mathrm{u}}-E_{1}\right) h^{2}}{24(1+v)}=B_{42}, \\
& C_{33}=\frac{\left(E_{\mathrm{u}}+E_{1}\right) h^{3}}{24(1-v)}, \quad C_{44}=\frac{\left(E_{\mathrm{u}}+E_{1}\right) h^{3}}{48(1+v)}=C_{22}
\end{aligned}
$$

The transverse shear stiffness can be estimated as

$$
\Gamma_{1}=\lambda^{2} \frac{A_{44} C_{22}-B_{42}^{2}}{A_{44}}=\frac{\lambda^{2}}{72} \frac{\left[3\left(G_{\mathrm{u}}+G_{1}\right)^{2}-\left(G_{\mathrm{u}}-G_{1}\right)^{2}\right] h^{3}}{G_{\mathrm{u}}+G_{1}}
$$

with $\lambda$ following from

$$
\frac{\mathrm{d}}{\mathrm{d} z}\left[G(z) \frac{\mathrm{d} Z}{\mathrm{~d} z}\right]+\lambda_{*}^{2} G(z) Z=0,\left.\quad \frac{\mathrm{d} Z}{\mathrm{~d} z}\right|_{|z|=\frac{h}{2}}=0
$$

Instead of $G(z)$ one has to take the second equation of (31). The solution of this spectral problem may be written by using Kummer functions [1]. Dependence $\Gamma_{1}$ versus $g_{1} \equiv \frac{G_{1}}{G_{\mathrm{u}}}$ is shown in the Fig. 2. It is easy to prove that a good approximation of this curve is given by

$$
\frac{\Gamma_{1}}{G_{\mathrm{u}} h^{3}}=\frac{\pi^{2}}{12}+0.416\left(1-g_{1}\right)
$$




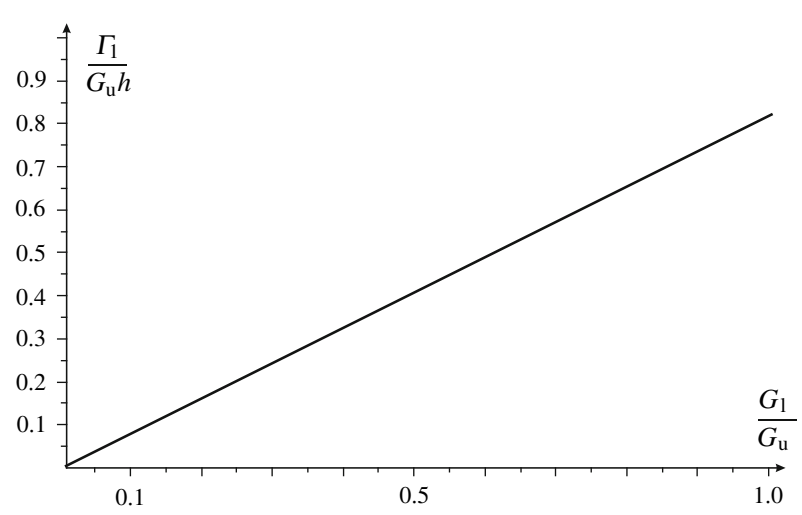

Fig. 2 Normalised transverse shear stiffness in dependence of the shear modulus

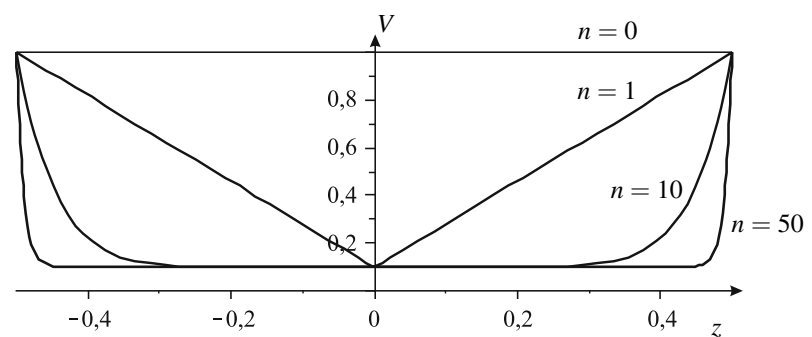

Fig. 3 Distribution of porosity

\subsubsection{Nonlinear symmetric distribution}

In this section, we consider the small deformations of a functionally graded plate made of a metallic or a polymer foam. For the panel made of a porous metallic foam the distribution of the pores over the thickness can be inhomogeneous. Let us introduce $h$ as the thickness of the panel, $\rho_{\mathrm{s}}$ as the density of the bulk material and $\rho_{\mathrm{p}}$ as the minimum value of the density of the foam. For the description of the symmetric distribution of the porosity, we assume the power law

$$
V(z)=\rho+(1-\rho)\left|\frac{2 z}{h}\right|^{n},
$$

where $\rho=\frac{\rho_{\mathrm{p}}}{\rho_{\mathrm{s}}}, n$ is the power. $n=0$ corresponds to the homogeneous plate, for $n=1$ we have the linear distribution of porosity through the thickness. The distribution can be established, for example, if the plate is made of two symmetric layers. If $n>1$ one has a more complex distribution. If $n \gg 1$ the plate core has an approximately constant porosity, but the distribution of the density in the face layers is significant inhomogeneous. Examples of the distribution are shown in Fig. 3 for $n=0,1,10,50$ with $\rho=0.1$.

The elastic properties of a metallic foam highly depend on the porosity and the cell structure. The dependence of Young's modulus and shear modulus on porosity is given by the power law $[9,12]$

$$
\frac{E_{\mathrm{p}}}{E_{\mathrm{S}}} \sim \rho^{m}, \quad \frac{G_{\mathrm{p}}}{G_{\mathrm{S}}} \sim \rho^{m}
$$

where $E_{\mathrm{p}}$ and $G_{\mathrm{p}}$ are the Young's modulus and shear modulus of the foam, respectively, $E_{\mathrm{s}}$ and $G_{\mathrm{s}}$ are the Young's modulus and shear modulus of material which is used to synthesize a foam. The value $m$ depends on the structure of the foam. For a closed-cell foam $m=1$, for an open-cell foam $m \approx 2$. For the isotropic case, the graphs of the normalized $A_{11}, A_{44}, C_{33}, C_{22}$ are given in Fig. 4. Here we used $m=2, \rho=0.1, v=0.3$.

To obtain the dependence of the transverse shear stiffness we have to solve Eq. (28). The solution of the spectral problem (28) was made numerically by using shooting method [29]. The values of normalized $\Gamma_{1}$ versus $n$ is given in Fig. 5. 

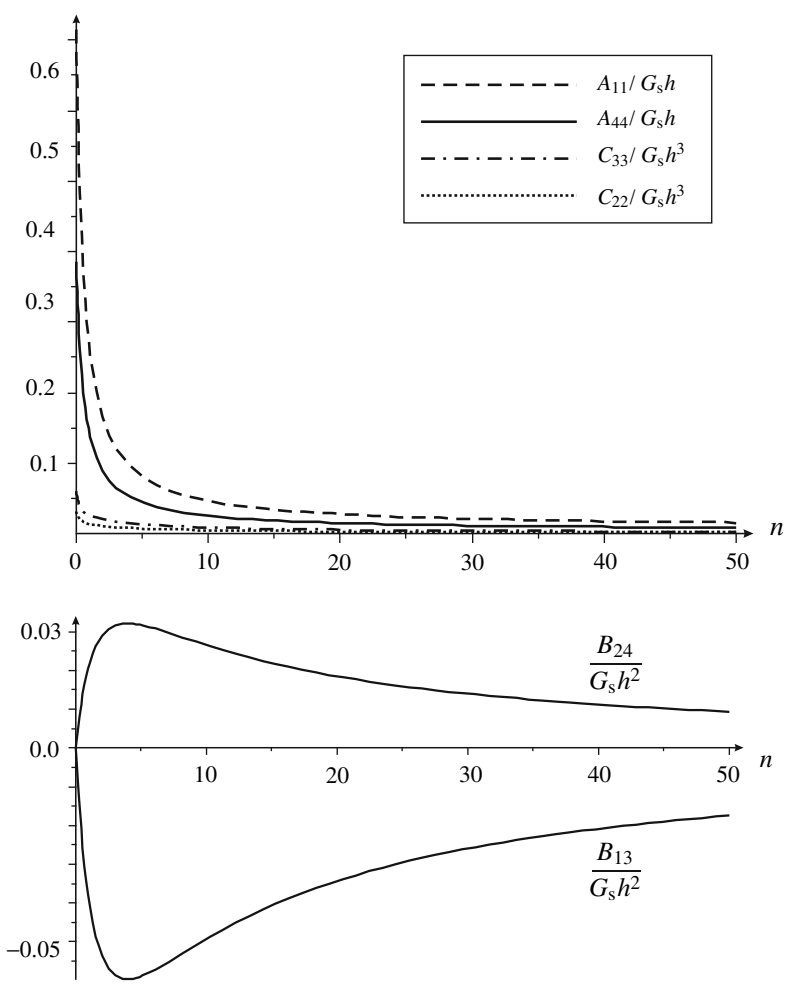

Fig. 7 Elastic moduli in dependence of the $n$-value

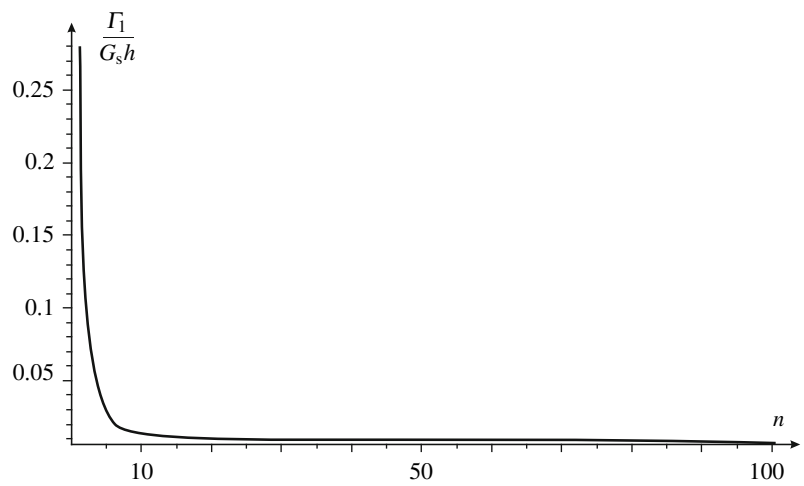

Fig. 8 Normalized transverse shear stiffness in dependence of the $n$-value

\section{Equations of symmetric orthotropic plate}

Let us consider the equilibrium equations of an orthotropic plate with a symmetric structure. In this case Eqs. (13) split into two parts: the plane problem for tangential displacements $u_{1}$ and $u_{2}$, and the bending problem for the $w, \varphi_{1}$ and $\varphi_{2}$, respectively.

The constitutive equations for an orthotropic symmetric plate can be given as follows

$$
\begin{aligned}
T_{1} & =\left(A_{11}+2 A_{12}+A_{22}\right) \mu_{1}+\left(A_{11}-A_{22}\right) \mu_{2}, \quad T_{2}=\left(A_{11}-A_{22}\right) \mu_{1}+\left(A_{11}-2 A_{12}+A_{22}\right) \mu_{2}, \\
T_{12} & =2 A_{44} \mu_{12}, \\
T_{1 n} & =\left(\Gamma_{1}+\Gamma_{2}\right) \gamma_{1}, \quad T_{2 n}=\left(\Gamma_{1}-\Gamma_{2}\right) \gamma_{2}, \\
M_{1} & =\left(C_{33}-2 C_{34}+C_{44}\right) \kappa_{1}+\left(C_{33}-C_{44}\right) \kappa_{2}, \quad M_{2}=\left(C_{33}-C_{44}\right) \kappa_{1}+\left(C_{33}+2 C_{34}+C_{44}\right) \kappa_{2} \\
M_{12} & =C_{22}\left(\kappa_{12}+\kappa_{21}\right) .
\end{aligned}
$$


In Cartesian coordinates with the geometrical relations (17) the equations

$$
T_{1,1}+T_{12,2}+q_{1}=0, \quad T_{12,1}+T_{2,2}+q_{2}=0
$$

are reduced to the form

$$
\begin{aligned}
& \left(A_{11}+2 A_{12}+A_{22}\right) u_{1,11}+\left(A_{11}-A_{22}\right) u_{2,21}+A_{44}\left(u_{1,21}+u_{2,11}\right)+q_{1}=0, \\
& \left(A_{11}-A_{22}\right) u_{1,12}+\left(A_{11}-2 A_{12}+A_{22}\right) u_{2,22}+A_{44}\left(u_{1,22}+u_{2,12}\right)+q_{2}=0
\end{aligned}
$$

The equation

$$
T_{1 n, 1}+T_{2 n, 2}+q_{n}=0
$$

has the following form

$$
\left(\Gamma_{1}+\Gamma_{2}\right) w_{, 11}+\left(\Gamma_{1}-\Gamma_{2}\right) w_{, 22}+\left(\Gamma_{1}+\Gamma_{2}\right) \varphi_{1,1}+\left(\Gamma_{1}-\Gamma_{2}\right) \varphi_{2,2}+q_{n}=0 .
$$

The equations

$$
M_{1,1}+M_{12,2}-T_{1 n}+m_{1}=0, \quad M_{12,1}+M_{2,2}-T_{2 n}+m_{2}=0
$$

result in

$$
\begin{aligned}
& \left(C_{33}-2 C_{34}+C_{44}\right) \varphi_{1,11}+\left(C_{33}-C_{44}\right) \varphi_{2,21}+C_{22}\left(\varphi_{1,22}+\varphi_{2,12}\right) \\
& \quad-\left(\Gamma_{1}+\Gamma_{2}\right) \varphi_{1}-\left(\Gamma_{1}+\Gamma_{2}\right) w_{, 1}+m_{1}=0, \\
& \left(C_{33}+2 C_{34}+C_{44}\right) \varphi_{2,22}+\left(C_{33}-C_{44}\right) \varphi_{1,12}+C_{22}\left(\varphi_{1,21}+\varphi_{2,11}\right) \\
& \quad-\left(\Gamma_{1}-\Gamma_{2}\right) \varphi_{2}-\left(\Gamma_{1}-\Gamma_{2}\right) w_{, 2}+m_{2}=0
\end{aligned}
$$

\subsection{One-dimensional case}

First, let us consider the bending of a rectangular plate when $q_{1}=0, q_{2}=0, q_{n}=q(x), m_{1}=m_{1}(x)$, $m_{2}=0$. Then we can assume that $u_{1}=u_{2}=0, w=w(x), \varphi_{1}=\varphi(x), \varphi_{2}=0$. Equation (39) reduces to

$$
\left(\Gamma_{1}+\Gamma_{2}\right) w^{\prime \prime}+\left(\Gamma_{1}+\Gamma_{2}\right) \varphi^{\prime}+q=0,
$$

where $(\ldots)^{\prime}$ denotes $(\ldots), 1$.

Equations (41) reduce to one equation

$$
\left(C_{33}-2 C_{34}+C_{44}\right) \varphi^{\prime \prime}-\left(\Gamma_{1}+\Gamma_{2}\right) \varphi-\left(\Gamma_{1}+\Gamma_{2}\right) w^{\prime}+m_{1}=0
$$

Let us transform Eqs. (42) and (43) to one equation with respect to $w$. After some manipulations we obtain

$$
\left(C_{33}-2 C_{34}+C_{44}\right) w^{\prime \prime \prime \prime}=q-\frac{\left(C_{33}-2 C_{34}+C_{44}\right)}{\Gamma_{1}+\Gamma_{2}} q^{\prime \prime}+m_{1}^{\prime}
$$

If we consider the other one-dimensional case when $q_{1}=0, q_{2}=0, q_{n}=q(y), m_{1}=0, m_{2}=m_{2}(y)$, then we can assume that $u_{1}=u_{2}=0, w=w(y), \varphi_{2}=\varphi(y), \varphi_{1}=0$. Then Eqs. (39) and (41) reduce to

$$
\begin{aligned}
& \left(\Gamma_{1}-\Gamma_{2}\right) w^{\prime \prime}+\left(\Gamma_{1}-\Gamma_{2}\right) \varphi^{\prime}+q_{n}=0, \\
& \left(C_{33}+2 C_{34}+C_{44}\right) \varphi^{\prime \prime}-\left(\Gamma_{1}-\Gamma_{2}\right) \varphi-\left(\Gamma_{1}-\Gamma_{2}\right) w^{\prime}+m_{2}=0
\end{aligned}
$$

where now (.)' denotes (.),2.

By using the same manipulations, we obtain the equation with respect to $w$

$$
\left(C_{33}+2 C_{34}+C_{44}\right) w^{\prime \prime \prime \prime}=q-\frac{\left(C_{33}+2 C_{34}+C_{44}\right)}{\Gamma_{1}-\Gamma_{2}} q^{\prime \prime}+m_{2}^{\prime}
$$

Equations (44) or (47) differ from one-dimensional variant of Eq. (1). If $m_{1}=0$ or $m_{2}=0$ and $\Gamma_{1}+\Gamma_{2} \rightarrow \infty$ or $\Gamma_{1}-\Gamma_{2} \rightarrow \infty$ one gets the Kirchhoff's plate equation with $D=C_{33}-2 C_{34}+C_{44}$ or with $D=$ $C_{33}+2 C_{34}+C_{44}$. 


\subsection{Two-dimensional case}

Let us eliminate the functions $\varphi_{1}$ and $\varphi_{2}$ from the Eqs. (39) and (41). For brevity, let us assume that $m_{1}=0$, $m_{2}=0$. By using operator notations, the Eqs. (41) may be rewritten in the form

$$
L_{11} \varphi_{1}+L_{12} \varphi_{2}=b_{1} w, \quad L_{21} \varphi_{1}+L_{22} \varphi_{2}=b_{2} w
$$

where

$$
\begin{array}{rlrl}
L_{11} & =\left(C_{33}-2 C_{34}+C_{44}\right) \partial_{1}^{2}+C_{22} \partial_{2}^{2}-\Gamma_{1}-\Gamma_{2}, & L_{22} & =\left(C_{33}+2 C_{34}+C_{44}\right) \partial_{2}^{2}+C_{22} \partial_{1}^{2}-\Gamma_{1}+\Gamma_{2}, \\
L_{21} & =L_{12}=\left(C_{33}+C_{22}-C_{44}\right) \partial_{1} \partial_{2}, & & \\
b_{1} & =\left(\Gamma_{1}+\Gamma_{2}\right) \partial_{1}, & b_{2} & =\left(\Gamma_{1}-\Gamma_{2}\right) \partial_{2}, \\
\partial_{\alpha}(\ldots) & \equiv(\ldots)_{, \alpha}, \quad \alpha=1,2 &
\end{array}
$$

From (48) we obtain the relations

$$
\mathcal{L} \varphi_{1}=\mathcal{L}_{1} w, \quad \mathcal{L} \varphi_{2}=\mathcal{L}_{2} w
$$

where

$$
\mathcal{L}=L_{11} L_{22}-L_{12}^{2}, \quad \mathcal{L}_{1}=L_{22} b_{1}-L_{12} b_{2}, \quad \mathcal{L}_{2}=L_{11} b_{2}-L_{21} b_{1}
$$

Using operator notations the Eq. (39) can be rewritten as follows

$$
\mathcal{L}_{w} w+b_{1} \varphi_{1}+b_{2} \varphi_{2}+q_{n}=0
$$

where

$$
\mathcal{L} w=\left(\Gamma_{1}+\Gamma_{2}\right) \partial_{1}^{2}+\left(\Gamma_{1}-\Gamma_{2}\right) \partial_{2}^{2}
$$

Then we obtain one differential equation of 6th order with respect to $w$

$$
\left(\mathcal{L} \mathcal{L}_{w}+b_{1} \mathcal{L}_{1}+b_{2} \mathcal{L}_{2}\right) w+\mathcal{L} q_{n}=0
$$

4.3 Two-dimensional case of an isotropic plate

For the isotropic plate we have that $C_{22}=C_{44}, C_{34}=0, \Gamma_{2}=0$. Then

$$
\begin{array}{ll}
L_{11}=\left(C_{33}+C_{44}\right) \partial_{1}^{2}+C_{44} \partial_{2}^{2}-\Gamma_{1}, & L_{22}=\left(C_{33}+C_{44}\right) \partial_{2}^{2}+C_{44} \partial_{1}^{2}-\Gamma_{1}, \\
L_{21}=L_{12}=C_{33} \partial_{1} \partial_{2}, & b_{2}=\Gamma_{1} \partial_{2}, \\
b_{1}=\Gamma_{1} \partial_{1}, &
\end{array}
$$

and

$$
\begin{aligned}
\mathcal{L} & =\left[\left(C_{33}+C_{44}\right) \partial_{1}^{2}+C_{44} \partial_{2}^{2}-\Gamma_{1}\right]\left[\left(C_{33}+C_{44}\right) \partial_{2}^{2}+C_{44} \partial_{1}^{2}-\Gamma_{1}\right]-C_{33}^{2} \partial_{1}^{2} \partial_{2}^{2}, \\
\mathcal{L}_{1} & =\Gamma_{1}\left[\left(C_{33}+C_{44}\right) \partial_{2}^{2}+C_{44} \partial_{1}^{2}-\Gamma_{1}\right] \partial_{1}+\Gamma_{1} C_{33} \partial_{1} \partial_{2}^{2}, \\
\mathcal{L}_{2} & =-\Gamma_{1} C_{33} \partial_{1}^{2} \partial_{2}+\Gamma_{1}\left[\left(C_{33}+C_{44}\right) \partial_{1}^{2}+C_{44} \partial_{2}^{2}-\Gamma_{1}\right] \partial_{2}
\end{aligned}
$$

Finally, the bending equation (49) has the form

$$
\left(C_{33}+C_{44}\right) \Delta \Delta w=\partial_{1} m_{1}+\partial_{2} m_{2}-\frac{C_{33}+C_{44}}{\Gamma_{1}} \Delta q_{n}+q_{n}
$$

If $m_{1}=m_{2}=0$ and $\Gamma_{1} \rightarrow \infty$ one gets the Kirchhoff's plate equation with $D=C_{33}+C_{44}$. 


\section{Bending of a plate made from functionally graded material}

In this case one gets a decoupling of the in-plane state and the plate state. Let us assume the Kirchhoff's plate bending. The governing equation (1) preserves the same form-only instead of the bending stiffness $D$ the effective bending stiffness with respect to (26) must be introduced as follows:

$$
D_{\text {eff }}=C_{33}+C_{44}
$$

and the external load $q$ should be replaced by $\partial_{1} m_{1}+\partial_{2} m_{2}-\frac{D_{\text {eff }}}{\Gamma_{1}} \Delta q_{n}+q_{n}$.

To analyze the influence of the transverse shear stiffness on the deflection of a plate let us consider a bending of a rectangular plate made of a functionally graded material. For brevity, let us assume that $m_{1}=m_{2}=0$, $x_{1} \in[0, a], x_{2} \in[0, b]$, where $a$ and $b$ are the length and width of the plate. Using the assumption $[9,12]$ that $v=$ const and that the Eqs. (26), (27) are valid, we can rewrite Eq. (50) in the following form:

$$
D_{\mathrm{eff}} \Delta \Delta w=q_{n}-\frac{2}{1-v} \frac{1}{\lambda^{2} h^{2}} \Delta q_{n}
$$

Introducing dimensionless variables by formulas

$$
\bar{w}=h^{-1} w, \quad \bar{x}_{1}=h^{-1} x_{1}, \quad \bar{x}_{2}=h^{-1} x_{2}, \quad \bar{x}_{1} \in\left[0, \frac{a}{h}\right], \quad \bar{x}_{2} \in\left[0, \frac{b}{h}\right], \quad \bar{q}=\frac{q_{n} h^{3}}{D_{\mathrm{eff}}},
$$

Equation (51) transforms to

$$
\Delta \Delta \bar{w}=\bar{q}-\frac{2}{1-v} \frac{1}{\lambda^{2} h^{2}} \Delta \bar{q}
$$

Here $\Delta=\frac{\partial^{2}}{\partial \bar{x}_{1}^{2}}+\frac{\partial^{2}}{\partial \bar{x}_{2}^{2}}$.

Let us consider sinusoidal load $\bar{q}=Q \sin \frac{\pi h \bar{x}_{1}}{a} \sin \frac{\pi h \bar{x}_{2}}{b}(Q=$ const $)$ and the boundary conditions of type (16). Then the solution of Eq. (52) is given by

$$
\bar{w}=\frac{K}{\eta^{2}} Q \sin \frac{\pi h \bar{x}_{1}}{a} \sin \frac{\pi h \bar{x}_{2}}{b}, \quad K=1+\frac{2 \eta}{1-v} \frac{1}{\lambda h^{2}}, \quad \eta=\left(\frac{\pi h}{a}\right)^{2}+\left(\frac{\pi h}{b}\right)^{2}
$$

For the Kirchhoff's plate theory $K=K_{\mathrm{K}} \equiv 1$, for the Mindlin's plate theory $K=K_{\mathrm{M}} \equiv 1+\frac{2 \eta}{1-v} \frac{1}{\pi^{2}}$, respectively. Let us consider the maximal deflection $W=\frac{K}{\eta^{2}} Q$. Thus, for the Kirchhoff's plate theory, we obtain that $W_{\mathrm{K}}=\frac{1}{\eta^{2}} Q$, for the Mindlin's plate theory we obtain that $W_{\mathrm{M}}=\frac{K_{\mathrm{M}}}{\eta^{2}} Q$, and in the general case $W_{\mathrm{F}}=\frac{K}{\eta^{2}} Q$, respectively.

Let us use following values $v=0.3, a=b, h=0.05 a$. Several normalized deflections are presented in the Table 1. It is easy to see that for the functionally graded plates the difference between theories may be significant. Let us note that for the cases of other types of boundary conditions, the influence of the structure of the plate on the deflection may be greater than for the used simple support type boundary conditions.

\section{Summary and outlook}

A plate theory based on Zhilin's direct approach method in the theory of shells for FGM is introduced. The basic items of the theory are related to

- the formulation of all balances for a deformable directed surface (a priori two-dimensional equations) and - the specific constitutive equation 
Table 1 Maximal deflections of quadratic plate and corresponding $\Gamma_{1}$

\section{Model}

$W / W_{\mathrm{K}}$

$\Gamma_{1} / G_{\mathrm{S}} h$

Kirchoff's plate

1

1.01

$\infty$

Mindlin's plate

FGM plate linear distribution (29)

$$
\begin{aligned}
& g_{\mathrm{m}}=0.5 \\
& g_{\mathrm{m}}=0.1 \\
& g_{\mathrm{m}}=0.05
\end{aligned}
$$

FGM plate power law (32)

$$
\begin{aligned}
& n=2 \\
& n=5 \\
& n=10
\end{aligned}
$$

In addition, for each class of plates the identification of the unknown material parameters should be performed. It is clearly shown that based on the assumption of linear elastic behavior the identification for different foams can be realized. In this case it was helpful that Zhilin's theory includes elements of symmetry groups for two-dimensional objects and the concept of effective properties. A simple static problem is solved and the solution is compared with a Kirchhoff-type theory and a Mindlin-type theory.

From the calculations one can conclude that for some problems the influence of the transverse shear is significant. The next step should be directed on the verification of this conclusion in the case of other boundary condition. Since it is well-known that, if the influence of the shear stiffness is significant, the rotatory inertia has also an influence, dynamical problems should be investigated. Last but not least the theory should be extended to the thermodynamical case.

\section{References}

1. Abramowitz, M., Stegun, I.: (eds.) Handbook of Mathematical Functions. Dover, New York (1972)

2. Altenbach, H.: Eine direkt formulierte lineare Theorie für viskoelastische Platten und Schalen. Ingenieur Archiv 58, 215228 (1988)

3. Altenbach, H.: An alternative determination of transverse shear stiffnesses for sandwich and laminated plates. Int. J. Solids Struct. 37(25), 3503-3520 (2000)

4. Altenbach, H.: On the determination of transverse shear stiffnesses of orthotropic plates. ZAMP 51, 629-649 (2000)

5. Altenbach, H., Zhilin, P.: A general theory of elastic simple shells (in Russian). Uspekhi Mekhaniki 11(4), 107-148 (1988)

6. Altenbach, H., Zhilin, P.A.: The theory of simple elastic shells. In: Kienzler, R., Altenbach, H., Ott, I. (eds.) Critical Review of the Theories of Plates and Shells and New Applications. Lect. Notes Appl. Comp. Mech, vol. 16, pp. 1-12. Springer, Berlin (2004)

7. Altenbach, H., Altenbach, J., Naumenko, K.: Ebene Flächentragwerke. Grundlagen der Modellierung und Berechnung von Scheiben und Platten. Springer, Berlin (1998)

8. Altenbach, H., Altenbach, J., Kissing, W.: Mechanics of Composite Structural Elements. Springer, Berlin (2004)

9. Ashby, M.F., Evans, A.G., Fleck, N.A., Gibson, L.J., Hutchinson, J.W., Wadley, H.N.G.: Metal Foams: A Design Guid. Butterworth-Heinemann, Boston (2000)

10. Banhart, J.: Manufacturing routes for metallic foams. J. Miner. 52(12), 22-27 (2000)

11. Chróścielewski, J., Makowski, J., Pietraszkiewicz, W.: Statics and dynamics of multifold shells. Non-linear theory and finite element method (in Polish). Wydawnictwo IPPT PAN, Warszawa (2004)

12. Gibson, L.J., Ashby, M.F.: Cellular Solids: Structure and Properties, 2nd edn. Cambridge Solid State Science Series. Cambridge University Press, Cambridge (1997)

13. Grigolyuk, E.I., Seleznev, I.T.: Nonclassical theories of vibration of beams, plates and shelles (in Russian). In: Itogi nauki i tekhniki, Mekhanika tverdogo deformiruemogo tela, vol. 5, VINITI, Moskva (1973)

14. Gupta, N., Ricci, W.: Comparison of compressive properties of layered syntactic foams having gradient in microballoon volume fraction and wall thickness. Mater. Sci. Eng. A 427, 331-342 (2006) 
15. El Hadek, M.A., Tippur, H.V.: Dynamic fracture parameters and constraint effects in functionally graded syntactic epoxy foams. Int. J. Solids Struc. 40, 1885-1906 (2003)

16. Kienzler, R.: On the consistent plate theories. Arch. Appl. Mech. 72, 229-247 (2002)

17. Kienzler, R., Altenbach, H., Ott, I.: (eds.) Critical review of the theories of plates and shells, new applications. Lect. Notes Appl. Comp. Mech. vol. 16, Springer, Berlin (2004)

18. Kirchhoff, G.R.: Über das Gleichgewicht und die Bewegung einer elastischen Scheibe. Crelles Journal für Die Reine Und Angewandte Mathematik 40, 51-88 (1850)

19. Libai, A., Simmonds, J.G.: The Nonlinear Theory of Elastic Shells, 2nd edn. Cambridge University Press, Cambridge (1998)

20. Lurie, A.I.: Theory of Elasticity. Foundations of Engineering Mechanics. Springer, Berlin (2005)

21. Mindlin, R.D.: Influence of rotatory inertia and shear on flexural motions of isotropic, elastic plates. Trans. ASME J. Appl. Mech. 18, 31-38 (1951)

22. Naghdi, P.: The theory of plates and shells. In: Flügge, S. (ed.) Handbuch der Physik, vol. VIa/2, pp. 425-640. Springer, Heidelberg (1972)

23. Nye, J.F.: Physical Properties of Crystals. Oxford Science Publications, Clarendon, Oxford (2000)

24. Reissner, E.: On the theory of bending of elastic plates. J. Math. Phys. 23, 184-194 (1944)

25. Reissner, E.: The effect of transverse shear deformation on the bending of elastic plates. J. Appl. Mech. 12(11), A69A77 (1945)

26. Reissner, E.: On bending of elastic plates. Q. Appl. Math. 5, 55-68 (1947)

27. Reissner, E.: Reflection on the theory of elastic plates. Appl. Mech. Rev. 38(11), 1453-1464 (1985)

28. Rothert, H.: Direkte Theorie von Linien- und Flächentragwerken bei viskoelastischen Werkstoffverhalten. Techn.-Wiss. Mitteilungen des Instituts für Konstruktiven Ingenieurbaus 73-2, Ruhr-Universität, Bochum (1973)

29. Stoer, J., Bulirsch, R.: Introduction to Numerical Analysis. Springer, New York (1980)

30. Timoshenko, S.P., Woinowsky-Krieger, S.: Theory of Plates and Shells. McGraw Hill, New York (1985)

31. Truesdell, C.: Die Entwicklung des Drallsatzes. ZAMM 44(4/5), 149-158 (1964)

32. Zhilin, P.A.: Mechanics of deformable directed surfaces. Int. J. Solids Struc. 12, 635-648 (1976)

33. Zhilin, P.A.: Applied mechanics. Foundations of the theory of shells (in Russian). St Petersburg State Polytechnical University (2007) 